\title{
Novel Survivable Logical Topology Routing in IP-over-WDM Networks by Logical Protecting Spanning Tree Set
}

\author{
Zhili Zhou*, Tachun Lin ${ }^{\dagger}$, Krishnaiyan Thulasiraman ${ }^{\ddagger}$, Guoliang Xue ${ }^{\S}$ and Sartaj Sahni ${ }^{\Uparrow}$ \\ *IBM Research Collaboratory, Singapore \\ ${ }^{\dagger}$ Cameron University, Lawton, OK, USA \\ ¥University of Oklahoma, Norman, OK, USA

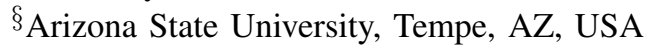 \\ TUniversity of Florida, Gainesville, FL, USA \\ Email: zhili@sg.ibm.com, tlin@cameron.edu, thulasi@ou.edu,xue@asu.edu, sahni@cise.ufl.edu
}

\begin{abstract}
The survivable logical topology mapping problem in an IP-over-WDM optical network is to map each link $(u, v)$ in the logical topology (at the IP layer) into a lightpath between the nodes $u$ and $v$ in the physical topology (at the optical layer) such that failure of a physical link does not cause the logical topology to become disconnected. It is assumed that both the physical and logical topologies are at least 2-edge connected. Generating a survivable routing is an NP-complete problem. For this problem two lines of investigations have been pursued in the literature: the mathematical programming based approach initiated by Modiano et al., and the structural approach initiated by Kurant and Thiran and pursued further by Thulasiraman et al. The mathematical programming approach is not scalable for large networks, though it gives considerable insight into certain important aspects of the problem. The structural approach requires contraction and expansion of logical graphs and computing link-disjoint lightpaths between pairs of nodes in the physical topology. In this paper, we propose a novel approach based on the concept of protecting spanning tree set of the logical topology. The basic idea is to identify a set of spanning trees of the logical topology and a routing of the logical links such that at least one of these trees remains connected after a physical link failure. Given a set of trees of the logical topology we first present three optimization problems with varying degrees of difficulty relating to this approach and discuss their Integer Linear Programming formulations. We then consider the general case when both the tree set and a survivable routing are to be determined. For this general case we present a heuristic approach. We incorporate in this heuristic a method to augment the logical topology with additional links to guarantee a survivable routing. This approach has several interesting features. It only requires a shortest path algorithm and an algorithm to generate appropriate spanning trees. It also provides a framework for generating a survivable routing for the SRLG failure case. Contractions of graphs and disjoint path generation are not required that greatly reduces the computation time. We provide results of extensive simulations conducted to evaluate our new approach.
\end{abstract}

\section{INTRODUCTION}

Wavelength-Division Multiplexing (WDM) technology is widely applied in long-haul (next-generation) networks for its high bandwidth and reliability. The communication between two end nodes on WDM networks is carried out through a path; namely a lightpath, which utilizes a single wavelength through optical nodes like OXCs and OADMs without optoelectro-optical (O-E-O) conversion on intermediate optical nodes. Most data services nowadays, like HTTP, VoIP, FTP, etc. apply a dominating protocol called IP. For an IP-overWDM network, the traffic on each IP link is carried through a lightpath in the WDM network. For a multi-hop data transmission in IP-over-WDM network as shown in Figure 1, the traffic on 1-2-4 path in the IP network is implemented through two lightpaths 1-2 and 2-3-4 in the WDM network. Given an
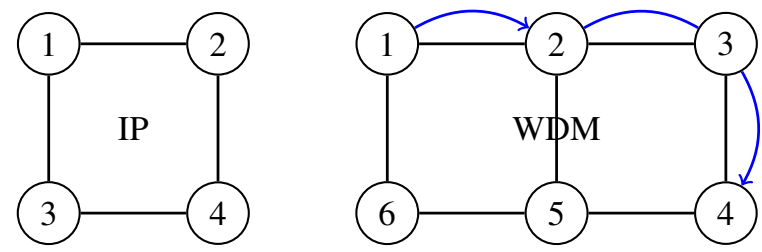

Fig. 1. The example of potential lightpaths for logical link

IP-over-WDM network with physical and logical topologies $G_{P}=\left(V_{P}, E_{P}\right), G_{L}=\left(V_{L}, E_{L}\right)$, where $V_{P}\left(V_{L}\right)$ represents physical (logical) nodes/vertices and $E_{P}\left(E_{L}\right)$ represents physical (logical) edges/links, a survivable routing in such a network is usually determined by the edge-disjoint lightpath routing for logical edges. If any physical link failure does not cause a cut in the logical topology, this routing is called a survivable routing. For this problem two lines of investigations have been pursued in the literature: the mathematical programming based approach initiated by Modiano and NarulaTam [1], and the structural approach initiated by Kurant and Thiran [2] and pursued further by Thulasiraman et al. [3][4]. The mathematical programming approach is not scalable for large networks, though it gives considerable insight into certain important aspects of the problem. The structural approach requires contraction and expansion of logical graphs and computing link-disjoint lightpaths between pairs of vertices in the physical topology.

In this paper, we propose a novel approach using multiple 
logical spanning trees and their corresponding lightpath routing to guarantee survivability. This idea was motivated by the general concept that an IP network will be survivable if there exists a logical spanning tree after any single link failure in the WDM network. Here we would like to find a set of logical spanning trees such that any physical failure will only cut off chords with respect to one or several spanning trees in the set.

The rest of this paper is organized as follows. In section II we review previous related research works. In section III we present several basic concepts and notations. Given a set of trees of the logical topology in section IV we present three optimization problems with varying degrees of difficulty relating to our approach and discuss their Integer Linear Programming (ILP) formulations. One of these problems is also shown to be equivalent to the minimum dominating set problem in bipartite graphs. In section $\mathrm{V}$ we present a heuristic for the general case when when both the tree set and a survivable routing are to be determined. We incorporate in this heuristic a method to augment the logical topology with additional links to guarantee a survivable routing. In section VI we evaluate this heuristic using extensive simulations. In section VII we conclude by pointing out some future extensions of our work.

\section{RELATED WORK}

Survivability of a logical topology mapping (routing) can be guaranteed if the lightpaths in the physical topology corresponding to this mapping are all link-disjoint. Since finding mutually disjoint paths between multiple pairs of nodes is NP-complete [5], survivable design of the logical topology in an IP-over-WDM network is also an NP-complete problem. Modiano and Narula-Tam [1] proved a necessary and sufficient condition for survivable routing under a single failure in IPover-WDM networks and formulated the problem as an ILP. Todimala and Ramamurthy [6] adapted the concept of Shared Risk Link Group introduced in [7] and also computed the routing through an ILP formulation. Extensions of the work in [1] are given in [8] [9]. [8] introduced certain connectivity metrics for layered networks and provided ILP formulations for the lightpath routing problem satisfying these metrics. In particular, they provided approximation heuristics for lightpath routing maximizing the min cross layer cut metric. This metric captures the robustness of the networks after multiple physical link failures. Kan et al. [9] discussed the relationship between survivable lightpath routing and the spare capacity requirements on the logical links to satisfy the original traffic demands after failures. A common drawback of ILP approaches is that they are not scalable as the network size increases. Hence, heuristic approaches that provide approximations to the optimal solutions are presented.

To handle the drawback of ILP approaches, Kurant and Thiran [2] proposed the Survivable Mapping by Ring Trimming (SMART) framework which first attempts to find linkdisjoint paths for the links of a subgraph of the given logical graph. If such mappings exist, the subgraph is contracted. The procedure is repeated until the logical graph is contracted to a single node, or at some step disjoint mappings cannot be found. In the former case, the resulting mappings define a survivable mapping of the given logical graph. In the latter case, they conclude that no survivable mapping of the given logical graph exists. Another approach proposed by Lee et al. [10] utilized the concept of ear-decomposition on biconnected topologies. One can show that this is, in fact, a special variant of the framework given in [2], though it was developed independently. Javed et al. obtained improved heuristics based on SMART [11]. Using duality theory in graphs, a generalized theory of logical topology survivability was given by Thulasiraman et al. [3][4]. Thulasiraman et al. [12] considered the problem of augmenting the logical graph with additional links to guarantee the existence of a survivable mapping. It has been shown in [12] that if the physical network is 3-edge connected, an augmentation of the logical topology that is guaranteed to be survivable is always possible. An earlier work that discussed augmentation is in [13].

\section{BASIC CONCEPTS AND NOTATIONS}

We let $G_{P}=\left(V_{P}, E_{P}\right)$ and $G_{L}=\left(V_{L}, E_{L}\right)$ represent the physical and logical networks, respectively. The relationship between $G_{P}$ and $G_{L}$ is that $V_{L} \subseteq V_{P}$. We let $e, f, g$ represent physical edges and $u, v$ represent logical edges. We let $i, j$ denote physical nodes and $s, t$ for the logical nodes. Edges and links as well as nodes and vertices will be used interchangeably.

Without loss of generality, we keep the indices of logical nodes same as their corresponding physical nodes. For a logical edge $u$ we find a path $p$ in the physical topology whose start and end nodes are the two corresponding nodes of $u$. We call $p^{u}$ the lightpath of $u$.

We let $i(u)$ and $j(u)$ be the physical nodes of logical edge $u$ and $s(e)$ and $t(e)$ be the logical nodes of physical edge $e$. If $u$ connects $s(u)$ and $t(u)$, then, $(s(u), t(u))=u$. The lightpath provides the routing for a logical edge. The failure of any physical edge in $p^{u}$ disconnects the lightpath of $u$ and its corresponding logical edge.

Definition 1: We define $\tau$ as a protecting spanning tree of the logical network, if it is a spanning tree in the logical network which remains connected after at least one physical link failure. $\tau^{C}$ is the co-tree of protecting spanning tree $\tau$ with $\tau^{C}=G_{L} \backslash \tau$

Definition 2: We define $\mathcal{T}$ as a protecting spanning tree collection, if its elements are protecting spanning trees in the logical network. With a given protecting spanning tree collection $\mathcal{T}$, we let $\mathcal{T}^{C}$ be a protecting spanning co-tree collection, if its elements are the co-trees of elements in the protecting spanning tree collection.

We now define the mapping and its corresponding co-mapping.

Definition 3: Mapping and co-mapping of substructure in the logical network:

1. Mapping $M$ maps each logical edge $v$ to physical edges by lightpath, that is, $M: v \rightarrow p^{v}$ with $v \in E_{L}$. (Note: Here $M(v)$ also stands for the edges in the path $p^{v}$.) 
2. Mapping $M$ maps protecting spanning tree $\tau$ to a subgraph of the physical network; that is, $M: \tau \rightarrow$ $\bigcup_{v \in \tau} M(v)$.

3. Mapping $M$ maps protecting spanning tree collection, $\mathcal{T}$, to a subgraph of the physical network, $M: \mathcal{T} \rightarrow$ $\bigcup_{\tau \in \mathcal{T}} M(\tau)$.

4. Co-mapping $M^{C}$ maps logical edge $v$ to a subgraph of the physical network, $M^{C}: v \rightarrow G_{P} \backslash M(v)$ with $v \in$ $E_{L}$.

5. Co-mapping $M^{C}$ maps protecting spanning tree $\tau$ to a subgraph of the physical network, $M^{C}: \tau \rightarrow G_{P} \backslash$ $\bigcup_{v \in \tau} M(v)$; that is, $M^{C}(\tau)=\bigcap_{v \in \tau} M^{C}(v)$

6. Co-mapping $M^{C}$ maps protecting spanning tree collection $\mathcal{T}$ to a subgraph of the physical network, $M^{C}: \mathcal{T} \rightarrow$ $\bigcup_{\tau \in \mathcal{T}}\left(G_{P} \backslash M(\tau)\right)$; that is, $M^{C}(\mathcal{T})=\bigcup_{\tau \in \mathcal{T}} M^{C}(\tau)$

Note: As in (1) in Definition 3, in all cases $M(\cdot)$ stands for all the edges in the corresponding subgraph.

Example 1: We illustrate the above definitions using Figure 2. $\tau_{1}=\{(1,2),(2,6),(6,4)\}, \tau_{2}=\{(6,1),(1,4),(4,2)\}$ are two protecting spanning trees in the logical network. The lightpath $p^{12}=\{(1,2)\}$, the lightpath $p^{26}=$ $\{(2,5),(5,6)\}$, the lightpath $p^{64}=\{(6,5),(5,4)\}$, the lightpath $p^{61}=\{(6,1)\}$, the lightpath $p^{14}=\{(1,4)\}$, and the lightpath $p^{42}=\{(4,3),(3,2)\}$. The mapping and co-mapping of logical edge $(2,6)$ are $p^{26}=$ $\{(2,5),(5,6)\}$ and $\{(1,2),(1,4),(1,6),(2,3),(3,4),(4,5)\}$, respectively. The mapping of the protecting spanning tree $\tau_{1}$, $M\left(\tau_{1}\right)=\left\{p^{12}, p^{26}, p^{64}\right\}=\{(1,2),(2,5),(4,5),(5,6)\}$. Its co-mapping is $M^{C}\left(\tau_{1}\right)=\{(1,4),(1,6),(2,3),(3,4)\}$. The mapping of $\tau_{2}, M\left(\tau_{2}\right)=\left\{p^{61}, p^{14}, p^{42}\right\}$, and its co-mapping is $M^{C}\left(\tau_{2}\right)=\{(1,2),(2,5),(4,5),(5,6)\} \cdot \mathcal{T}=\left\{\tau_{1}, \tau_{2}\right\}$ is a protecting spanning tree collection. The mapping of this collection $M(\mathcal{T})=E_{P}$ and its co-mapping $M^{C}(\mathcal{T})=E_{P}$.

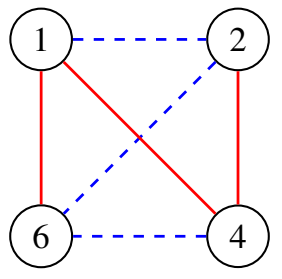

Logical network

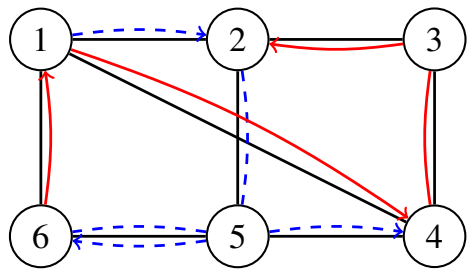

Physical network
Fig. 2. The lightpath, spanning tree, and mapping example

Note that the relationship between the mapping of logical edge, protecting spanning tree $\tau$, and the protecting spanning tree collection $\mathcal{T}$ is as follows:

1. $M(\tau)=\bigcup_{v \in \tau} M(v)=\bigcup_{v \in \tau} p^{v}$.

2. $M(\mathcal{T})=\bigcup_{\tau \in \mathcal{T}} M(\tau)=\bigcup_{v \in \tau, \tau \in \mathcal{T}} M(v)=$ $\bigcup_{v \in \tau, \tau \in \mathcal{T}} p^{v}$.

Consider a routing of all the logical links in an IP-overWDM network. Let $\mathcal{T}$ be a collection of spanning trees in the logical network. If a physical link $(i, j)$ is in the set $M^{C}(\tau)$ for some spanning tree $\tau \in \mathcal{T}$ then the failure of $(i, j)$ does not disconnect $\tau$ and so the logical network will remain connected after the failure of $(i, j)$.

Definition 4: If physical link $(i, j)$ is in the set $M^{C}(\tau)$ for some spanning tree $\tau$ in a given collection $\mathcal{T}$ of spanning trees then $\tau$ is said to protect $(i, j)$. If for every physical link $(i, j)$, there exists a tree in a collection $\mathcal{T}$ which protects $(i, j)$, then the routing is a survivable routing.

Definition 5: Given a routing of the logical network, the set $\left[\mathcal{T}, M(\mathcal{T}), M^{C}(\mathcal{T})\right]$ where $\mathcal{T}$ is a protecting tree set is survivable if $M^{C}(\mathcal{T})=E_{P}$. If $M^{C}(\mathcal{T}) \neq E_{P}$ then the set $\left[\mathcal{T}, M(\mathcal{T}), M^{C}(\mathcal{T})\right]$ is partially survivable.

We demonstrate an example of partial survivability in a given IP-over-WDM network as follows.

Example 2: Following Example 1, let $\mathcal{T}=\left\{\tau_{1}\right\}$. Then a single failure in physical edges $(1,4),(1,6),(2,3)$, and $(3,4)$ not utilized by $\tau_{1}$ does not disconnect logical tree branches $(1,2),(2,6)$, and $(6,4)$. Hence the logical network is connected. Therefore, $\left[\mathcal{T}, M(\mathcal{T}), M^{C}(\mathcal{T})\right]$ is partially survivable with $M(\mathcal{T})=\{(1,2),(2,5),(4,5),(5,6)\}$ and $M^{C}(\mathcal{T})=$ $\{(1,4),(1,6),(2,3),(3,4)\}$.

\section{Protecting Spanning Tree Set Optimization AND ILP FORMULATIONS}

In this section we present three optimization problems with different levels of difficulty relating to protecting tree set selection for survivability. For each problem we present and discuss an ILP formulation. For all these optimization problems we assume that a collection $\mathcal{T}$ of spanning trees of the logical graph is given. We shall consider the general case without this assumption in section $\mathrm{V}$.

We let $G_{P}=\left(V_{P}, E_{P}\right)$ and $G_{L}=\left(V_{L}, E_{L}\right)$ be the physical and logical networks and $\mathcal{T}$ be a tree collection, which is a logical spanning tree set.

First, we introduce variables which would be used in the following formulations:

$x_{i j}^{\ell}$ : binary variable, indicates whether the logical network is protected by spanning tree $\ell$ after $(i, j)$ failure

$y_{\ell}$ : binary variable, whether spanning tree $\ell$ is selected

$z_{i j}^{s t}$ : binary variable, whether $(s, t)$ is routed through $(i, j)$

$\beta_{i j}^{\ell}$ : binary variable, whether $(i, j)$ is protected by a selected spanning tree $\ell$

$g_{i j}$ : binary variable, equals 0 if physical link $(i, j)$ failure causes the disconnection of logical network

\section{A. Minimum Protecting Spanning Tree Set Problem (MPTS)}

Given a collection $\mathcal{T}$ of spanning trees of the logical graph, and assuming that there exists a survivable routing under which all the physical links are protected by the trees in $\mathcal{T}$, the Minimum Protecting Spanning Tree Set (MPTS) is to determine a routing of all the logical links that minimizes the cardinality of the subset of $\mathcal{T}$ that protects all the physical links. The following is an ILP formulation of the MPTS problem. 
MPTS:

$$
\begin{aligned}
& \min _{y} \sum_{\ell \in \mathcal{T}} y_{\ell} \\
& \text { s.t. } \quad \sum_{(i, j) \in E_{P}} z_{i j}^{s t}-\sum_{(j, i) \in E_{P}} z_{j i}^{s t}=\left\{\begin{array}{c}
1, \text { if } s=i,(s, t) \in E_{L} \\
-1, \text { if } t=i,(s, t) \in E_{L} \\
0, \text { otherwise }
\end{array}\right. \\
& z_{i j}^{s t}+z_{j i}^{s t} \leq 1-x_{i j}^{\ell}, \quad(s, t) \in \ell, \ell \in \mathcal{T},(i, j) \in E_{P} \\
& \beta_{i j}^{\ell} \leq x_{i j}^{\ell}+x_{j i}^{\ell}, \quad \ell \in \mathcal{T},(i, j) \in E_{P} \\
& \beta_{i j}^{\ell} \leq y_{\ell}, \quad \quad \ell \in \mathcal{T},(i, j) \in E_{P} \\
& \sum_{\ell \in \mathcal{T}} \beta_{i j}^{\ell} \geq 1, \quad(i, j) \in E_{P} . \\
& y_{\ell}, z_{i j}^{s t}, \beta_{i j}^{\ell}, x_{s t}^{\ell}, \ell \in \mathcal{T},(s, t) \in E_{L},(i, j) \in E_{P}
\end{aligned}
$$

We now discuss each equation/constraint in the ILP for the MPTS problem.

Route (Lightpath) Selection: Equation (1) selects a lightpath for each logical link $(s, t)$ using flow conservation principle. $z_{i j}^{s t}=1$ if $(s, t)$ is routed through physical link $(i, j)$; otherwise, $z_{i j}^{s t}=0$. Constraint (2) relates $z_{i j}^{s t}$ and $x_{i j}^{\ell}$ where $x_{i j}^{\ell}=1$ if tree $\ell$ protects physical link $(i, j)$, and is zero, otherwise.

$x_{i j}^{\ell}=0$ if a logical link $(s, t) \in \ell$ is routed through physical link (i,j) (that is, $z_{i j}^{s t}+z_{j i}^{s t}=1$ ) then constraint (2) forces $x_{i j}^{\ell}=0$. If no logical link $(s, t) \in \ell$ is routed through physical link $(i, j)$ then $x_{i j}^{\ell}=1$.

Protecting Property of Selected Spanning Trees: We let variable $\beta_{i j}^{\ell}$ indicate whether physical link $(i, j)$ is protected by a selected spanning tree $\ell$ or not. The value of $\beta_{i j}^{\ell}$ must satisfy the following:

i) If $x_{i j}^{\ell}+x_{j i}^{\ell}=1$ and $y_{\ell}=1$, then, $\beta_{i j}^{\ell}=0$ or 1 .

ii) If $x_{i j}^{\ell}+x_{j i}^{\ell}=1$ and $y_{\ell}=0$, then, $\beta_{i j}^{\ell}=0$.

iii) If $x_{i j}^{\ell}+x_{j i}^{\ell}=0$ and $y_{\ell}=1$, then, $\beta_{i j}^{\ell}=0$;

iv) If $x_{i j}^{\ell}+x_{j i}^{\ell}=0$ and $y_{\ell}=0$, then, $\beta_{i j}^{\ell}=0$;

The above requirements will be satisfied by constraints (3), (4), and (5).

To guarantee that each $(i, j)$ is protected by a selected spanning tree (that is, a tree $\ell$ for which $y_{\ell}=1$ ), we need

$$
\sum_{\ell \in \mathcal{T}}\left(x_{i j}^{\ell}+x_{j i}^{\ell}\right) y_{\ell} \geq 1, \quad(i, j) \in E_{P} .
$$

This is a non-linear inequality. To linearize this, consider the following.

$$
\begin{aligned}
& \sum_{\ell \in \mathcal{T}}\left(x_{i j}^{\ell}+x_{j i}^{\ell}\right) y_{\ell} \\
\geq & \sum_{\ell \in \mathcal{T}} \beta_{i j}^{\ell}, \text { because of (3) and (4) } \\
= & \sum_{\ell \in \mathcal{T},\left(x_{i j}^{\ell}+x_{j i}^{\ell}\right)=1 \text { and } y_{\ell}=1} \beta_{i j}^{\ell}, \text { because } \beta_{i j}^{\ell}=0, \\
& \text { when }\left(x_{i j}^{\ell}+x_{j i}^{\ell}\right)=0 \text { or } y_{\ell}=0 \\
\geq & 1, \text { because of }(5) .
\end{aligned}
$$

Thus, constraints (3)-(5) guarantee that $\sum_{\ell \in \mathcal{T}}\left(x_{i j}^{\ell}+x_{j i}^{\ell}\right) y_{\ell} \geq 1$, for $(i, j) \in E_{P}$. In other words, constraints (3)-(5) provide the survivable condition under any physical link failure. Thus constraints (3)-(5) guarantee that the trees selected (that is, those trees $\ell$ for which $y_{\ell}=1$ ) protect all the physical links.

\section{B. Minimum Protecting Spanning Tree Set and Maximum Link Protection Problem (MPTS-MaxLP)}

Given a collection $\mathcal{T}$ of spanning trees of the logical graph, Minimum Protecting Tree Set and Maximum Link Protection Problem (MPTS-MaxLP) is to determine a routing of all the logical links that minimizes the cardinality of the subset of $\mathcal{T}$ that protects the largest number of physical links.

Let a pair $(\Gamma, \Phi)$ correspond to a routing of the logical links if $\Phi$ is the cardinality of a subset of $\mathcal{T}$ that protects $\Gamma$ physical links under the routing. A solution to the MPTSMaxLP problem gives the pair $\left(\Gamma_{\max }, \Phi_{\min }\right)$ that has the property

$$
\Gamma_{\max } \geq \Gamma \text { and } \Phi_{\min } \leq \Phi
$$

for any $(\Gamma, \Phi)$ for the given collection of logical spanning trees.

This problem can be solved using a 2-stage approach as follows. In the following, $g_{i j}=1$ if after the failure of physical link $(i, j)$ at least one selected logical spanning tree remains connected; otherwise, $g_{i j}=0$.

Stage 1 . With a given protecting spanning tree collection $\mathcal{T}$, determine $\max \sum_{(i, j) \in E_{P}} g_{i j}=M$

Stage 2. Determine a minimum subset of the spanning tree set such that $\sum_{(i, j) \in E_{P}} g_{i j} \geq M$.

The following are the formulations for these two stages:

Stage 1:

$$
M=\max _{g} \sum_{(i, j) \in E_{P}} g_{i j}
$$

s.t. Constraints (1), (2), and (6)

$$
\begin{array}{ll}
g_{i j} \in\{0,1\}, & (i, j) \in E_{P} \\
\sum_{\ell \in \mathcal{T}} x_{i j}^{\ell} \geq g_{i j}, & (i, j) \in E_{P}
\end{array}
$$

Stage 2:

$$
\min _{y} \sum_{\ell \in \mathcal{T}} y_{\ell}
$$

s.t. Constraints (1), (2), (3), (4), (5), (6), and (7),

$$
\begin{aligned}
& \sum_{(i, j) \in E_{P}} g_{i j} \geq M \\
& \sum_{\ell \in \mathcal{T}} \beta_{i j}^{\ell} \geq g_{i j}, \quad(i, j) \in E_{P}
\end{aligned}
$$




\section{Minimum Dominating Set and the MPTS Problem}

In the two optimization problems considered we seek a routing of the logical links that achieves certain objectives. A special case of the MPTS problem is to find a minimum protecting tree set given a collection $\mathcal{T}$ of spanning trees as well as a routing. We refer to this problem as MPTS-S. Note that in the MPTS-S problem, the values of $z_{i j}^{s t}$ are known and so the ILP formulation of this problem is obtained by removing equation 1 from the ILP formulation given in section IV-A for the MPTS problem. This is given below.

$$
\begin{aligned}
& \min \sum_{\ell \in \mathcal{T}} y_{\ell} \\
& \text { s.t. constraints (2), (3)-(6) }
\end{aligned}
$$

We can also give a graph theoretic formulation using the concept of dominating set. Given an undirected graph $G=$ $(V, E)$, a node $v$ is said to dominate node $w$ if $v$ is adjacent to $v$. A subset $S$ of $V$ is a dominating set of $G$ if every vertex $u \in V-S$ is adjacent to a vertex in $S$. A dominating set with minimum cardinality is called a minimum dominating set of $G$.

Given a collection $\mathcal{T}$ of the spanning trees of a logical graph and a routing of the logical links, let $G$ be a bipartite graph with bipartition $(X, Y)$. Let each node in $X$ represent a tree in $\mathcal{T}$ and each node in $Y$ represent a physical link. Let edge $(u, v) \in E$ if and only if the tree corresponding to $u \in X$ protects the physical link represented by the node $v \in Y$. Then it can be seen that a minimum dominating set of $G$ is a solution for the MPTS-S problem.

\section{A Survivable Routing Algorithm Based on the SPANNING TreE Set: THE General CASE}

In this section, we propose a survivable routing algorithm based upon a spanning tree set. This algorithm produces a survivable routing as well as the protecting spanning tree set. We first introduce some extra notations for the algorithm. Let $P M$ be the collection of protecting spanning trees and their corresponding lightpath routing; i.e., $P M=\{(\tau, M(\tau))\}$. Let $Q M=\{(e, Q(e))\}, Q(e)=\{u: e \in M(u), e \in$ $\left.E_{P}, u \in E_{L}\right\}$; i.e., $Q M$ is a collection of physical edges and their corresponding logical edges whose lightpaths are routed through these physical edges. Let $w(e)$ be the weight on physical edge $e, w(u)$ be the weight on logical edge $u$, and $w(\tau)=\sum_{u \in \tau} w(u)$ be the weight of $\tau$. $\alpha$ and $\beta$ are the penalty functions used to adjust the weight of physical and logical links.

Algorithm 1 is comprised of two parts: generating a protecting spanning tree set and routing, and logical network augmentation. The weight on logical and physical edges are initialized to be 1 and the first logical spanning tree and the corresponding lightpaths for tree branches are generated. Following that, the weight of tree branches in selected logical spanning tree $\tau$ and the weight of physical edges on the lightpaths of $\tau$ are both increased. $\tau$ and its lightpath routing, $(\tau, M(\tau))$, is then stored in $P M$ and $M^{C(i)}(\mathcal{T})$ is updated with physical edges not utilized by $\tau$.
Note that the purpose of assigning weights to logical links is to avoid generating new logical spanning trees with edges which are already in the spanning tree set. We also assign weights to the physical links such that lightpaths for unmapped branches in the newly selected logical spanning tree would also avoid utilizing the same physical links in existing lightpaths.

After increasing the weight, the algorithm picks a minimum weight logical spanning tree which has at least an unmapped logical edge $u$ and generates its lightpath $p^{u}$. The path is generated using a shortest path algorithm. The above procedure repeatedly selects spanning trees and generates lightpaths till each logical edge has a designated lightpath.

For each tree in the spanning tree collection, there exists physical edges not utilized by the routing of tree branches. In other words, the failure of these physical edges will not disconnect the spanning tree. Hence, the lightpath routing is survivable if the union of unutilized physical edges of all trees in the tree collection is $E_{P}$; otherwise, there exists a physical edge whose failure will disconnect all trees in the tree collection. In the latter case, logical augmentation plays an important role to guarantee survivability. We use Figure 3 to illustrate the logical augmentation method.

Given Figure 3 as the physical topology, let the initial routing of logical edge $u$, where $i(u)=1$ and $j(u)=8$, be $M(u)=\{(1,4),(4,5),(5,8)\}$. Since an edge-disjoint path $p^{\widetilde{u}}$ to $p^{u}$ does not exist, two edge-disjoint paths for $u, p^{u}=\{(1,2),(2,3),(3,5),(5,8)\}$ and $p^{\widetilde{u}}=$ $\{(1,4),(4,6),(6,7),(7,8)\}$ are required to protect $u$ from failure. In other words, we need to add two parallel logical edges with $p^{u}, p^{\widetilde{u}}$ as their routing.

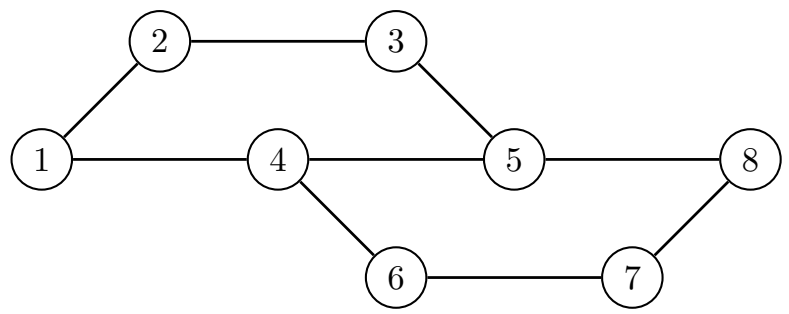

Fig. 3. Physical topology used to illustrate the logical augmentation method

With a given logical link $u$ and its lightpath $p^{u}$, we have two types of mapping for logical augmentation: (1) single augmentation: we augment logical link $u$ with parallel link $\widetilde{u}$ and assign $p^{\widetilde{u}}$ as a lightpath of $\widetilde{u}$ if there exists an edge-disjoint path $p^{\widetilde{u}}$ to $p^{u}$; and (2) double augmentation: if an edge-disjoint lightpath of $p^{u}$ does not exist, then we augment two logical links $u^{1}$ and $u^{2}$ parallel to $u$ and generate two edge-disjoint paths connecting $i(u)$ and $j(u)$ as their lightpaths, which are used to replace the original logical link and its lightpath routing.

\section{Simulation Results}

In this section, we present simulation results shown in Table I, which demonstrate the efficiency of the protecting spanning tree algorithm, Algorithm 1. The notations, $P N(L N)$, 


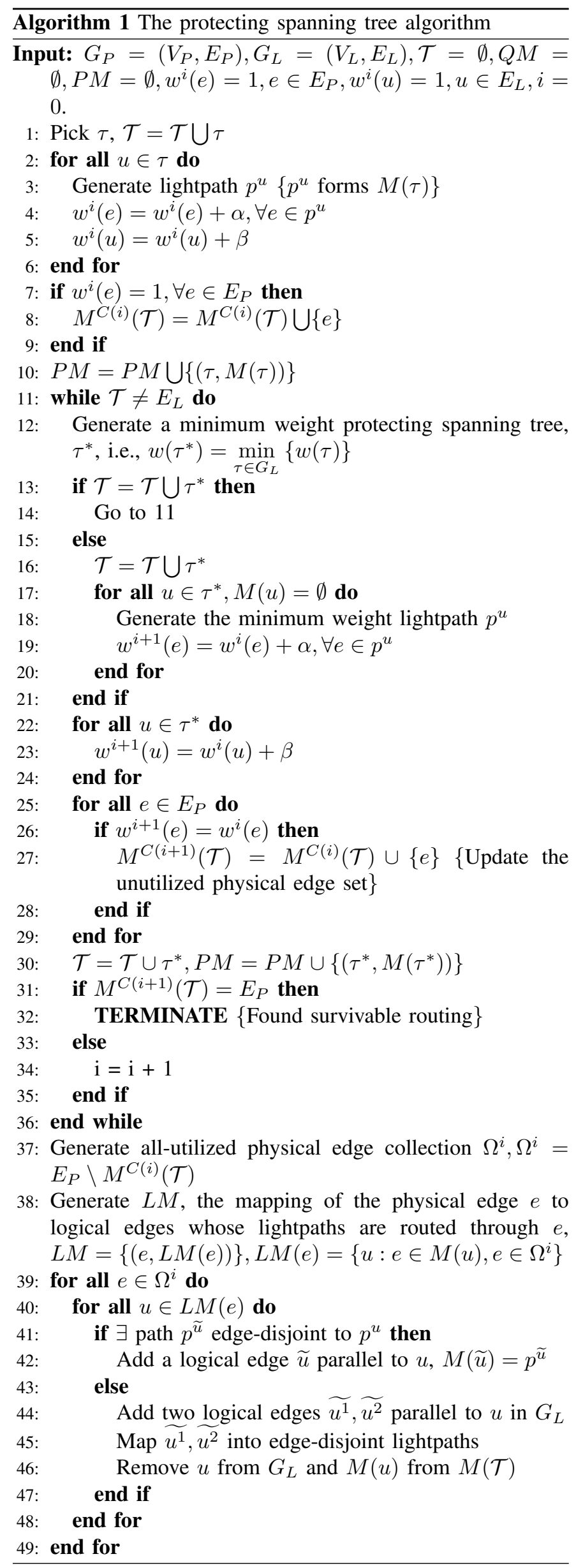

$P C(L C)$, and $P E(L E)$ used in Table I denote the number of physical (logical) nodes, connectivity of physical (logical) networks, and the number of physical (logical) edges, respectively. Survivability index, Surv\%, represents the percentage of physical edges whose failure do not disconnect the logical network. AugLog denotes the average number of augmented logical edges to guarantee survivability of each physicallogical topology pair; and TreeNo represents the average number of spanning trees generated by Algorithm 1.

In the simulation, we first generated random physical topologies, where the number of physical nodes were 10,20 , $\ldots$, or 50 and the topologies had connectivity $3,4,5$, or 6 . The logical topologies corresponding to the physical topologies had $(0.5 * \mid$ physical nodes $\mid)$ number of nodes and we let the logical connectivity to be $3, \ldots$, or |physical connectivity|. We generated 300 physical and logical networks for each setting $(P N, L N, P C, L C)$, applied our algorithm, and put the average in Table I.

Analyzing the results shown in the tables we have the following observations. Firstly, the survivability index showed that a very high percentage of physical link failure would not disconnect the logical network with the lightpath routing generated by Algorithm 1. Second, the simulation results show that a survivable routing is achieved with only a small number of trees. In most cases only a small number of link augmentations, $A u g L o g$, is required to guarantee a survivable routing. Surv\% indices close to $100 \%$ are denoted as $\approx 100$.

\section{CONCLUSION}

In this paper, we proposed a novel approach to the survivable logical topology mapping problem in an IP-over-WDM optical network based on the concept of protecting logical spanning tree set. Given a set of trees of the logical topology we first presented three optimization problems with varying degrees of difficulty relating to this approach and discussed their ILP formulations. We then considered the general case when both the tree set and a survivable routing are to be determined. For this general case we present a heuristic approach. We incorporate in this heuristic a method to augment the logical topology with additional links to guarantee a survivable routing. We presented simulation results to demonstrate the efficiency of this new approach.

The new approach has several interesting features. It only requires a shortest path algorithm and an algorithm to generate appropriate spanning trees. An algorithm such as the one in [14] that generates spanning trees one at a time in a simple and elegant manner is an appropriate candidate for use in this approach. Though this spanning tree generation algorithm was not used in Algorithm 1, incorporating it in Algorithm 1 will be an interesting future direction of research.

Moreover, the approach identifies a group of spanning trees of the logical graph and a lightpath routing of the logical links. Each tree is identified with a group of physical edges such that failure of one or more of these edges will leave at least one of the trees remains connected, guaranteeing survivability of the logical topology against these group failures. Thus the 


\begin{tabular}{|c|c|c|c|c|c|c|c|c|}
\hline PN & LN & $\mathrm{PC}$ & $\mathrm{LC}$ & $\mathrm{PE}$ & $\mathrm{LE}$ & Surv\% & AugLog & TreeNo \\
\hline \multirow[t]{7}{*}{10} & \multirow[t]{7}{*}{5} & 3 & 3 & 15 & 8 & 99.9996 & 3.16667 & 3.14667 \\
\hline & & 4 & 3 & 20 & 8 & 99.9999 & 3 & 3.17333 \\
\hline & & 4 & 4 & 20 & 10 & 100 & 0 & 3 \\
\hline & & 5 & 3 & 25 & 8 & 100 & 0 & 3.14333 \\
\hline & & 5 & 4 & 25 & 10 & 100 & 0 & 3 \\
\hline & & 6 & 3 & 30 & 8 & 100 & 0 & 3.13667 \\
\hline & & 6 & 4 & 30 & 10 & 100 & 0 & 3 \\
\hline \multirow[t]{10}{*}{20} & \multirow[t]{10}{*}{10} & 3 & 3 & 30 & 15 & 99.9954 & 3.04839 & 5.31333 \\
\hline & & 4 & 3 & 40 & 15 & 99.9988 & 2.95238 & 5.20333 \\
\hline & & 4 & 4 & 40 & 20 & 100 & 0 & 3.08667 \\
\hline & & 5 & 3 & 50 & 15 & 99.9999 & 3 & 5.33 \\
\hline & & 5 & 4 & 50 & 20 & 100 & 0 & 3.09333 \\
\hline & & 5 & 5 & 50 & 25 & 100 & 0 & 3.53 \\
\hline & & 6 & 3 & 60 & 15 & $\approx 100$ & 3 & 5.30333 \\
\hline & & 6 & 4 & 60 & 20 & 100 & 0 & 3.07333 \\
\hline & & 6 & 5 & 60 & 25 & 100 & 0 & 3.49667 \\
\hline & & 6 & 6 & 60 & 30 & 100 & 0 & 3.07667 \\
\hline \multirow[t]{10}{*}{30} & \multirow[t]{10}{*}{15} & 3 & 3 & 45 & 23 & 99.9916 & 2.99123 & 6.21333 \\
\hline & & 4 & 3 & 60 & 23 & 99.9972 & 3.21569 & 6.16333 \\
\hline & & 4 & 4 & 60 & 30 & 99.9997 & 4.85714 & 3.05 \\
\hline & & 5 & 3 & 75 & 23 & 99.9997 & 3 & 6.17667 \\
\hline & & 5 & 4 & 75 & 30 & 100 & 0 & 3.06667 \\
\hline & & 5 & 5 & 75 & 38 & 100 & 0 & 3.24333 \\
\hline & & 6 & 3 & 90 & 23 & 99.9999 & 3 & 6.17 \\
\hline & & 6 & 4 & 90 & 30 & 100 & 0 & 3.06333 \\
\hline & & 6 & 5 & 90 & 38 & 100 & 0 & 3.25 \\
\hline & & 6 & 6 & 90 & 45 & 100 & 0 & 3.07667 \\
\hline \multirow[t]{10}{*}{40} & \multirow[t]{10}{*}{20} & 3 & 3 & 60 & 30 & 99.9863 & 2.87838 & 9.5 \\
\hline & & 4 & 3 & 80 & 30 & 99.996 & 3.26923 & 9.02333 \\
\hline & & 4 & 4 & 80 & 40 & 99.9998 & 5.35714 & 3.03333 \\
\hline & & 5 & 3 & 100 & 30 & 99.9994 & 3.01887 & 9.32667 \\
\hline & & 5 & 4 & 100 & 40 & $\approx 100$ & 4 & 3.03333 \\
\hline & & 5 & 5 & 100 & 50 & 100 & 0 & 3.22333 \\
\hline & & 6 & 3 & 120 & 30 & 99.9997 & 2.83333 & 9.34667 \\
\hline & & 6 & 4 & 120 & 40 & $\approx 100$ & 4 & 3.04667 \\
\hline & & 6 & 5 & 120 & 50 & 100 & 0 & 3.20333 \\
\hline & & 6 & 6 & 120 & 60 & 100 & 0 & 3.04 \\
\hline \multirow[t]{10}{*}{50} & \multirow[t]{10}{*}{25} & 3 & 3 & 75 & 38 & 99.9829 & 3.02161 & 9.37 \\
\hline & & 4 & 3 & 100 & 38 & 99.9952 & 3.54713 & 9.54 \\
\hline & & 4 & 4 & 100 & 50 & 99.9999 & 6.69231 & 3.05333 \\
\hline & & 5 & 3 & 125 & 38 & 99.9995 & 3.26786 & 9.27667 \\
\hline & & 5 & 4 & 125 & 50 & $\approx 100$ & 4 & 3.02333 \\
\hline & & 5 & 5 & 125 & 63 & 100 & 0 & 3.15333 \\
\hline & & 6 & 3 & 150 & 38 & 99.9996 & 3.06 & 9.36333 \\
\hline & & 6 & 4 & 150 & 50 & $\approx 100$ & 4 & 3.03333 \\
\hline & & 6 & 5 & 150 & 63 & $\approx 100$ & 7 & 3.16333 \\
\hline & & 6 & 6 & 150 & 75 & 100 & 0 & 3.04333 \\
\hline
\end{tabular}

TABLE I

COMPUTATIONAL RESULTS

algorithm provides a framework for generating a survivable routing for the SRLG (Shared Risk Link Group) failure case.

The simulation results show that a survivable routing is achieved with only a small number of trees. In most cases only a small number of link augmentations is required to guarantee a survivable routing.

We believe that the approach along with mathematical programming [1] and structural [2][3][4] approaches provide several insights into the survivable logical topology routing problem in an IP-over-WDM optical network.

\section{ACKNOWLEDGEMENT}

Krishnaiyan Thulasiraman's, Guoliang Xue's, and Sartaj Sahni's research has been supported by NSF Grant Numbers 1115130, 1115129, and 1115184, respectively.

\section{REFERENCES}

[1] E. Modiano and A. Narula-Tam, "Survivable routing of logical topologies in WDM networks," in IEEE INFOCOM, 2001.

[2] M. Kurant and P. Thiran, "On survivable routing of mesh topologies in IP-over-WDM network," in IEEE INFOCOM, 2005.

[3] K. Thulasiraman, M. S. Javed, and G. Xue, "Circuits/Cutsets duality and a unified algorithmic framework for survivable logical topology design in IP-over-WDM optical networks," in IEEE INFOCOM, 2009.

[4] K. Thulasiraman, M. Javed, and G. Xue, "Primal meets dual: A generalized theory of logical topology survivability in IP-over-WDM optical networks," in Second International Conference on Communication Systems and Networks, 2010.

[5] R. M. Karp, "Reducibility among combinatorial problems," in Complexity of Computer Computations, 1st ed., R. E. Miller and J. W. Thatcher, Eds. Plenum Press, 1972, pp. 85-103.

[6] A. Todimala and B. Ramamurthy, "A scalable approach for survivable virtual topology routing in optical WDM networks," IEEE Journal on Selected Areas in Communications, vol. 25, pp. 63-69, 2007.

[7] J. Strand, A. L. Chiu, and R. Tkach, "Issues for routing in the optical layer," IEEE Communications Magazine, vol. 39, pp. 81-87, 2001.

[8] K. Lee and E. Modiano, "Cross-layer survivability in WDM-based networks," in IEEE INFOCOM, 2009.

[9] D. D. Kan, A. Narula-Tam, and E. Modiano, "Lightpath routing and capacity assignment for survivable IP-overWDM networks," in 7th International Workshop on Design of Reliable Communication Networks, 2009.

[10] S. Lee, J. Liu, and Y. Chen, "An ear-decomposition based approach for survivable routing in WDM networks," in 19th International Conference on Advanced Information Networking and Applications, 2005.

[11] M. Javed, K. Thulasiraman, and G. Xue, "Lightpaths routing for single link failure survivability in IP-overWDM networks," Journal of Communication and Networks, 2007.

[12] K. Thulasiraman, T. Lin, M. Javed, and G. Xue, "Logical topology augmentation for guaranteed survivability under multiple failures in IP-over-WDM optical networks," Optical Switching and Networking, pp. 206-214, 2010.

[13] C. Liu and L. Ruan, "A new survivable mapping problem in IP-over-WDM networks," IEEE Journal on Selected Areas in Communications, vol. 25, pp. 25-34, 2007.

[14] R. Jayakumar, K. Thulasiraman, and M. N. S. Swamy, "Complexity of computation of a spanning tree enumeration algorithm," IEEE Transactions on Circuits and Systems, pp. 853-860, October 1984. 